\title{
RISK PERCEPTION OF UNCERTAINTIES IN SUPPLY CHAIN
}

\begin{abstract}
The objective of this paper is to clarify the risk perception of uncertainties in supply chain under environmental turbulence. Rapid spread of globalization pushes firms to face the higher level of uncertainty, where firms must formulate necessary and sufficient strategy for supply chain management. Such a strategy, however, involves a broad range of factors, including some that are subjective. This paper addresses this issue by refining existing structural model of supply disruption. Interviews were conducted to demonstrates the applicability of the proposed structural model to the real markets in both developed and developing countries. The results clarify the difference in risk perception and provide managers with suggestion on how to formulate strategy for their supply chain management.
\end{abstract}

Keywords: supply chain management, supply disruption, risk perception, uncertainty.

\section{Introduction and Brief Literature Review}

With rapid spread of globalization, firms are to face the higher level of uncertainty, which increases the risk of supply disruption. Supply networks now span multiple geographic regions, increasing the exposure of local firms to environmental and operational risk. For example, the Great East Japan Earthquake in 2011 interrupted local and global distribution channels. The disaster resulted in shortages of critical components across every industry, in addition to the severe damage to life-line logistics network. Supply disruption is defined as "unplanned and unanticipated event that disrupt the normal flow of goods and materials within a supply chain and, as a consequence, expose firms within the supply chain to operational and financial risks" (Craighead et al., 2007), while from the perspective of managers responsible for supply chain operations, Ellis et al. (2010) defined supply disruption risk as "an individual's perception of the total potential loss associated with the disruption of supply of a particular purchased item from a particular supplier."

Despite the significant amount of research in the management techniques of supply disruption risk (e.g., Ellis et al., 2010; Tse et al., 2016), the environmental uncertain factors that impact on supply disruption have received only limited attention. Among these literatures, Tse et al. (2016) focused on the Thai beverage industries and explored the relationship of perception of demand, quality and logistics uncertainties and the representation of supply disruption risk. They further defined the concept of "overall supply disruption risk" that was represented by both magnitude and probability of supply disruption risks. The magnitude of supply disruption risk is given by the severity of the impact experienced by the supply network because of the incident; the probability of supply disruption risk is defined as the perceived likelihood that the normal flows of goods in downstream levels will be interrupted. Because of the lack of supply chain research in rapidly developing economies (Behara et al., 2014), focusing upon the Thai beverage industry provides an appropriate context for the exploration of complex supply issues.

On the other hand, as Kristal et al. (2011) demonstrated, the effectiveness of different supply chain practices is affected by national culture. Therefore, it is important to test the generalizability of the findings to other countries and across other industries. This paper 
addresses this research gap by focusing on the supply disruption risk of beverage industries that engage in business both in developed and developing countries. Based on the previous work (Tse et al., 2016), a refined model of supply disruption risk is proposed. The model considers the relationship of perception of abovementioned three uncertainties and overall disruption risk that may affect the practitioner's perception of disruption risk as well as the strategy for supply chain management.

\section{Research Design}

To structure uncertainty, Tse et al., (2016) conceptualized supply uncertainties as three uncontrollable factors in supply chain environments: demand, quality and logistics uncertainties. Demand uncertainty is associated with the predictability of product demand (Lee, 2002) and has been found to have a direct impact on the supply chain process variability (Germain et al., 2008), which stems from mismatching between the forecast by a company and actual demand and poor coordination to suppliers. Quality uncertainty is another area of concern for many organizations that makes them undertake inspection of incoming goods to establish the standard of received products. The uncertainty can cause a cascading effect through a supply network until issues reach the final consumer (Giunipero and Eltantawy, 2004). Logistics uncertainty is viewed as a factor that causes a delay or an interruption originating from logistics partners or natural disasters during the transportation process to customer, which can be caused by labor disputes, terrorist activities, natural disasters and infrastructure failures transportation (Chopra and Sodhi, 2004; Wilson, 2007; Lockamy III, 2014). The case of the Great East Japan Earthquake corresponds to this uncertainty.

One of the most difficult aspects in the analysis of supply disruption risk is how to deal with subjective risk perception and uncertainties among supply chain, which affects the strategy for supply chain management. To address the research gap of the original approach, the perception of the uncertainties is directly evaluated through the AHP. In addition to the approach to the perception of the uncertainties, the impact of the magnitude and the probability of risks to overall supply disruption risk are also analyzed through the AHP. Figure 1 illustrates the refined model of supply disruption risk, by which the relationship of perception of the uncertainties and overall supply disruption risk are analyzed.

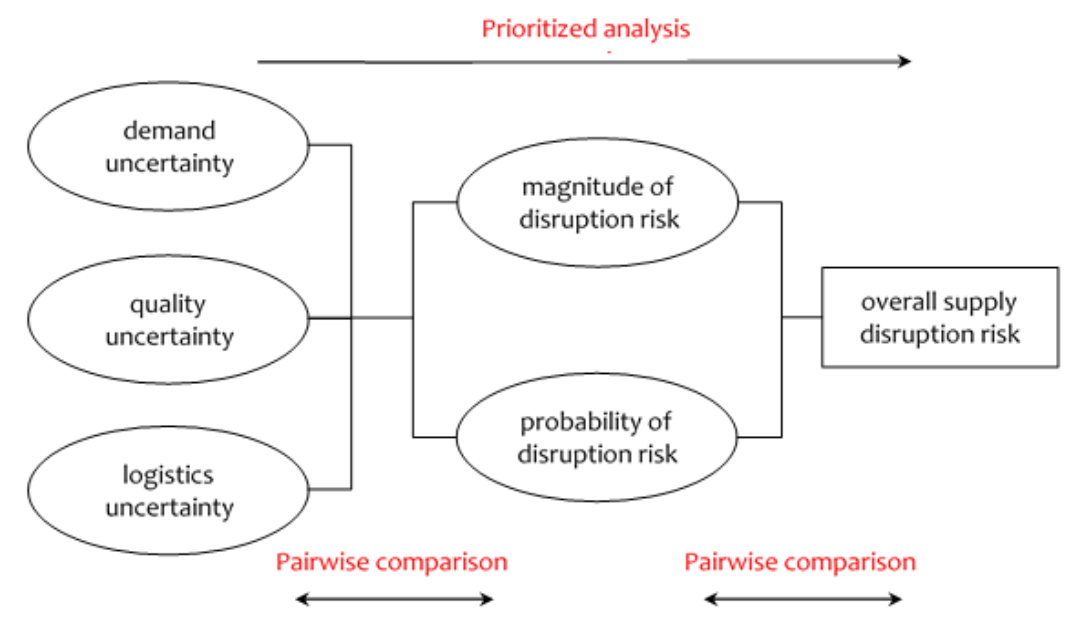

Fig. 1. Refined model of supply disruption risk. 
In the process of collecting information of practitioner's perception of disruption risk, interviews were carried out in beverage companies with a manager of developed-countries division and with another manager of developing-countries division. Both were responsible for supply chain operations in a beverage company, each of which engages in business both in developed and developing countries. In this paper, the practitioner's perception of disruption risk is supposed to be represented upon the uncertainties and upon the magnitude and the probability of disruption risks, including factors that are subjective and relate to overall disruption risk.

\section{Model Analysis}

The model analysis is based on clarifications of the relationship of: perception between, the uncertainties and the magnitude or the probability of disruption risks, that between, the risks and overall disruption risk. These evaluations are complicated since they require to estimate uncontrollable factors, and may be different in developed and developing countries. Let $\left(a_{i j}^{k}\right)(i, j=$ demand, quality, and logistics uncertainties; $k=1,2, \ldots, 10)$ denote a pairwise comparison matrix generated by a manager in Company $k$, who was responsible for developed countries. The matrix, $\left(a_{i j}^{k}\right)$, quantifies the relationship of perception between the uncertainties and the risks. Further let $\left(a_{p q}^{k}\right)(p, q=$ magnitude and probability of disruption risk; $k=1,2, \ldots, 10)$ denote a pairwise comparison matrix representing the relationship of perception between the risks and overall disruption risk, generated by the manager. Pairwise comparison matrices of developing countries, denoted by $\left(b_{i j}^{k}\right)$ and $\left(b_{p q}^{k}\right)$, were also generated by another manager in the company, who oversaw supply chain operation in developing countries. Leaving out the details here, all the Consistency Indices of pairwise comparison matrices are sufficiently small. The degree of significance of the uncertainties to overall disruption risk was calculated by taking the weighted sum of the outputs of $\left(a_{i j}^{k}\right)$ and $\left(a_{p q}^{k}\right)$ for developed countries and $\left(b_{i j}^{k}\right)$ and $\left(b_{p q}^{k}\right)$ for developing countries. The results show that manager's risk perception varies from company to company, and the most significant uncertainty to overall disruption risk are often different in the same company between markets, i.e., developed and developing countries.

To explain the difference in risk perception among the beverage companies, the companies are then characterized by following seven properties: sales, ordinary income, net income, net asset, profit ratio, equity ratio, and number of employees, in 2016. Among these properties, the correlation coefficients among ordinary income, net income and net asset are greater than 0.9, therefore, net income is employed as a representative of these three properties in the analyses. Based on the abovementioned five descriptions, principal features of beverage companies are elicited through principal component analysis. Extracted principal components of the descriptions can clarify the characteristics of each beverage company with scatter diagram. The distribution of the companies according to the principal components illustrates how each company perceived relationship between the uncertainties and overall disruption risk. The analysis employs the outputs of pairwise comparisons generated by the managers, not-normalized by $l_{1}$-norm. Leaving out the details, cumulative proportion of the top three components reaches $90.4 \%$; therefore, these three components, $\mathrm{C}_{m}(m=1, \ldots, 3)$ are used to represent characteristics of ten beverage companies. Table 1 shows the component score of each description.

As shown in Table 1, each component has following features: component $1\left(\mathrm{C}_{1}\right)$ is significantly positive about net income and profit ratio, and is neutral with equity ratio; component $2\left(\mathrm{C}_{2}\right)$ is significantly positive about sales and number of employee, and is neutral with profit ratio; component $3\left(\mathrm{C}_{3}\right)$ is significantly positive about equity ratio. This 
paper, therefore, defines each component as follows; $\mathrm{C}_{1}$ : profitability of a company; $\mathrm{C}_{2}$ : size of a company; $\mathrm{C}_{3}$ : stability of a company. Figures 2 and 3 are scatter diagrams of the ten beverage companies plotted on $\mathrm{C}_{1} \mathrm{C}_{2}$-plane and $\mathrm{C}_{1} \mathrm{C}_{3}$-plane, respectively. In the diagram, each marker represents a beverage company comprised of a set of two letters showing the perception of the uncertainties to overall disruption risk. The left (right) letter in black (in red) of a maker represents the most significant uncertainty to overall disruption risk in developed (developing) countries for a company. Each letter means: $D$ for demand, $Q$ for quality, and $L$ for logistics uncertainties.

Table 1. Component score

\begin{tabular}{cccccc}
\hline component & sales & net income & profit ratio & equity ratio & employee \\
\hline 1 & 0.2228 & 0.9455 & 0.8267 & 0.0389 & -0.3802 \\
\hline 2 & 0.9285 & 0.1104 & -0.0439 & -0.2134 & 0.7014 \\
\hline 3 & -0.1718 & -0.1221 & 0.3956 & 0.9124 & 0.5491 \\
\hline
\end{tabular}

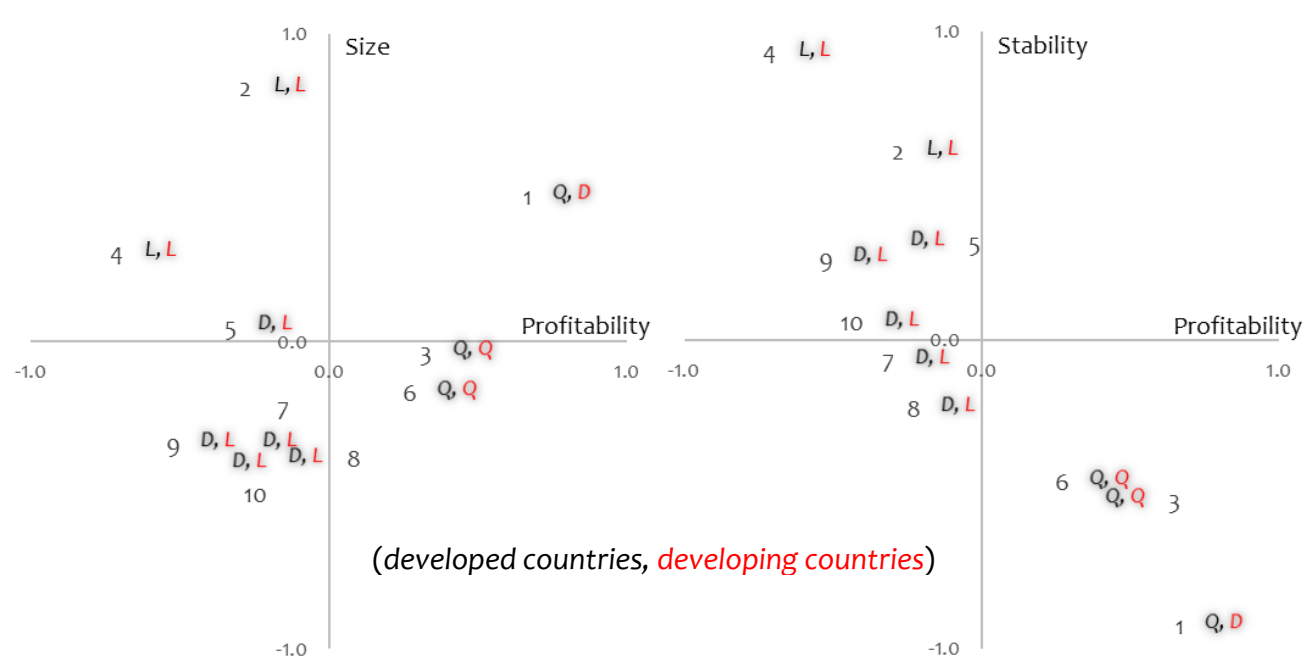

Fig. 2. Distribution on $\mathrm{C}_{1} \mathrm{C}_{2}$-plane.

Fig. 3. Distribution on $\mathrm{C}_{1} \mathrm{C}_{3}$-plane.

Concerning developed countries shown in Fig. 2, beverage companies perceiving demand uncertainty $(D)$ to be the most significant to overall disruption risk $(k=5,7,8,9$, $10)$ are plotted in the $2^{\text {nd }}$ and $3^{\text {rd }}$ quadrants. This result implies that small-to-medium-sized companies with rather low profitability consider that the perturbation of demand in market would seriously affect their supply chain. On the other hand, companies perceiving quality uncertainty $(Q)$ to be the most important factor to overall disruption risk $(k=1,3,6)$ distribute around high-profitability area. This means that profitable companies give greater importance to the quality of their products. As for companies perceiving logistics uncertainty $(L)$ to be the most significant to overall disruption risk $(k=2,4)$ are plotted in the $2^{\text {nd }}$ quadrant. The result implies that large companies with low profitability emphasize the importance of their logistic-network stability. On the other hand, in developing countries, companies perceiving $L$ to be the most significant to overall disruption risk $(k=2$, $4,5,7,8,9,10)$ are plotted in the $2^{\text {nd }}$ and $3^{\text {rd }}$ quadrants. This result implies that companies with rather low profitability consider that they need to maintain robust logistic network regardless of the size of a company. Moderately-sized companies with high profitability $(k=3,6)$ perceive $Q$ to be the most important to overall disruption risk. This means that such companies place greater importance on the quality of their products. In addition, large 
and profitable company $(k=1)$ perceives $D$ to be the most significant to overall disruption risk. This result implies that the company may have already maintained robust logistics network and may have confidence in its products quality; the remaining concern in business in developing countries is the perturbation of demand in market for the company.

As for developed countries shown in Fig. 3, the degree of stability of a company may affect the risk perception of beverage companies. Companies with high stability $(k=2,4)$ perceive $L$ to be the most significant to overall disruption risk, and companies with moderate stability $(k=5,7,8,9,10)$ consider $D$ to be the most important factor. On the other hand, companies with low stability $(k=1,3,6)$ perceive $Q$ to be the most significant to overall disruption risk. These results can be interpreted as follows: stable companies give greater importance to maintaining robust logistic network, and companies with moderate stability consider that the perturbation of demand in market would seriously affect their supply chain, unstable companies emphasize the importance of their product's quality. While in developing countries, it is the same as the case of developed countries that the degree of stability of a company features risk perceptions of the companies. Companies whose stability range from high to moderate $(k=2,4,5,7,8,9,10)$ place greater importance on maintaining robust logistic network, companies with low stability $(k=3,6)$ consider their product's quality to be important, and a company whose stability is the lowest among ten companies (while, its profitability is the highest) $(k=1)$ is conscious about the perturbation of demand in market.

\section{Concluding Remarks and Future Researches}

This paper clarifies the relationship of risk perception of uncertainties in supply chain and overall supply disruption risk. The differences in the degree of significance of the uncertainties among companies, or that between different markets suggest how each company or each division of a company should change its strategy for supply chain management when the perception of uncertainties would vary. Since each company has different feature in business, such as business policy, the proposed model can help managers to reflect their risk perception to the formulation of better strategy. Nonetheless, the proposed approach has some limitations. Further research needs to explore the following issues: the pros and cons of targeting relative significance in risk perception, the identification of new antecedents of supply disruption risk, and verification of the relationship between a company's risk perception and its actual behavior.

\section{Key References}

- Behara, R.S., Sunil B., Philip A.S. (2014). Leadership in OM research: a social network analysis of European researchers. Int. J. Oper. Prod. Man., 34(12), 1537-1563.

- Craighead, C.W., Jennifer B.M. Johnny R., Robert B.H. (2007). The Severity of Supply Chain Disruptions: Design Characteristics and Mitigation Capabilities. Decision Sci., 38(1), 131-156.

- Ellis, S.C., Raymond M.H., Jeff S. (2010). Buyer perceptions of supply disruption risk: A behavioral view and empirical assessment. J. Oper. Manag., 28(1), 34-46.

- Kristal, M., Mark P., Chenlung Y., Chwen S. (2011). Are supply chain management theories culturally constrained? An empirical assessment. Oper. Manage. Res., 4(1), 6173.

- Tse, Y.K., Matthews, R., Tan, K.H., Sato, Y., Pongpanich, C. (2016). Unlocking Supply Chain Disruption Risk within the Thai Beverage Industry. Ind. Manage. Data Syst., 116(1), 21-42. 